\title{
Pure laparoscopic pancreatoduodenectomy with initial approach to the superior mesenteric artery
}

\author{
Juan Santiago Azagra, Luca Arru, Sergio Estévez, Makkai-Popa Silviu-Tiberiu, Virginie Poulain, Martine Goergen \\ Centre Hospitalier de Luxembourg, Service de Chirurgie Générale et Mini-invasive, Luxembourg City, Luxembourg GD
}

Videosurgery Miniinv 2015; 10 (3): 450-457

DOI: $10.5114 /$ wiitm.2015.54040

\begin{abstract}
Introduction: The "artery-first approach" (AFA) to the superior mesenteric artery allows an early assessment of resectability of pancreatic tumours and could improve the benefits of laparoscopy, reducing invasiveness, especially for unresectable tumours.

Aim: To describe our technique of pure laparoscopic pancreatoduodenectomy (PLPD) with the AFA, and to report the surgical outcomes of this procedure in a small series of 12 patients through a retrospective analysis of a prospectively collected database.

Material and methods: Twelve selected patients underwent elective full laparoscopic pancreatoduodenectomy with the AFA. The technical aspects of the procedure are described in detail and the included images facilitate the understanding of the procedure.

Results: The mean operative time was $300 \mathrm{~min}$ (range: 250-540 min). No intraoperative complications were observed. No conversion to laparotomy was necessary. The mean postoperative hospital stay was 18 days (range: 8-42). Mortality was null. There were 3 major complications at the 3rd post-operative month follow-up: 2 patients reporting a grade $A$ pancreatic fistula and one biliary fistula.

Conclusions: Our work shows that pure laparoscopic pancreatoduodenectomy (PLPD) with the AFA is feasible, in selected patients. The AFA could improve on the advantages of laparoscopy in the identification of unresectable patients, and it also allows early control of vascular structures.
\end{abstract}

Key words: pancreatoduodenectomy, artery first approach, pure laparoscopy, feasibility, short-term outcomes, laparoscopy.

\section{Introduction}

In the last two decades, since the first laparoscopic pancreatoduodenectomy (LPD) was described in 1994 by Michel Gagner, the technique of pancreatoduodenectomy (PD) has been continuously evolving [1].

Current literature considers LPD a reliable and safe technique for selected patients [2], but also indicates that conversion rates to laparotomy remain high across different reports, varying between 10\% to $40 \%$ [3-5].
The main difficulties of LPD are related to the vascular dissection, and thus the major controversies concern the assessment of resectability and implicitly the selection of patients.

Classically, in open surgery, venous resection is well accepted and should be performed, to achieve RO resection, when it can be done safely. In contrast, resection of the superior mesenteric artery (SMA) is reported to increase postoperative morbidity and mortality rates without a remarkable improvement in survival, which meant that this radical approach has been largely abandoned $[6,7]$.

\section{Address for correspondence}

Luca Arru MD, Centre Hospitalier de Luxembourg, Service de Chirurgie Générale et Mini-Invasive, 4 Rue Barblé St, 1210 Luxembourg City, Luxembourg GD, phone: +35244112085, e-mail: lucaarrumd@gmail.com 
Besides, it is well known that laparoscopic staging allows identification of metastatic disease, unrecognised on the preoperative computed tomography $(\mathrm{CT})$ study, in about $10 \%$ of patients, sparing these individuals a laparotomy, without compromising the oncological prognosis and being accompanied by a low morbidity [8-11].

In light of this, the artery-first approach (AFA) to SMA can also be useful to assess the para-aortic nodal involvement. The presence of nodal metastasis at this level should prompt the surgeon to abandon a curative intent, except in the case of neuroendocrine tumours [12]. Furthermore, laparoscopic staging with the AFA could improve the intraoperative staging in the early stages of the procedure, before an irreversible step has been accomplished [13]. Also, during open surgery, the AFA is reported to reduce intraoperative blood loss and postoperative complications when compared with standard PD. However, none of the studies reports the number of resections abandoned owing to SMA invasion [14-18].

\section{Aim}

The aim of the article is to describe our technique of pure laparoscopic pancreatoduodenectomy (PLPD) with the AFA, and to report the surgical outcomes of this procedure in a small series of $12 \mathrm{pa}$ tients through a retrospective analysis of a prospectively collected database.

\section{Material and methods}

\section{Patient selection}

A series of 12 patients were selected to undergo PLPD with the AFA, according to strict inclusion and exclusion criteria. The study was accepted by all patients, who signed an exhaustive informed consent form.

The inclusion criteria we used were as follows:

- tumour located in the head of the pancreas or in the duodenum;

- resectable tumour: resectability was evaluated by pre-operative spiral CT with virtual angiography in order to exclude vascular involvement, nodal and distant metastasis;

- intention to treat by PLPD with the AFA.

The exclusion criteria applied for the selection of the study group were the following:
- pre-operative evidence of vascular invasion, requiring open procedure to safely carry out the superior mesenteric vein (SMV) dissection/resection; - peritoneal or hepatic metastasis;

- impossibility to achieve an $\mathrm{RO}$ resection without multi-visceral resection.

For the purpose of our study pancreatic fistula was defined and classified according to the recommendations of the International Study Group of Pancreatic Fistula (ISGPF) [19]. Delayed gastric emptying (DGE) was defined, for the purpose of our study, as the inability to return to a standard diet by the end of the first postoperative week according to the definition proposed by the International Study Group for Pancreatic Surgery (ISGPS) [20].

\section{Procedure}

The patient is placed in the supine position with the legs in abduction. Under general anaesthesia, the pneumoperitoneum is induced at a pressure of $14 \mathrm{~mm} \mathrm{Hg}$ using a Veress needle (Ethicon Endo-Surgery Inc., Cincinnati, $\mathrm{OH}$ ) and the trocars are inserted under direct vision. The position of the surgeons and the placement of the trocars depend on the stage of the procedure, as shown in Figure 1.

\section{Kocher manoeuvre}

The surgeons and patient are placed as shown in Figure $1 \mathrm{~A}$. The AFA technique that we employ was previously described by Partensky et al. for open surgery [12]. The absence of metastatic disease is confirmed by a complete laparoscopic exploration.

The procedure begins with the dissection of the hepatic flexure of the colon, which is mobilized and reflected downward (Photos 1.1-1.4), in order to obtain a wide Kocher manoeuvre, extended to the left side of the aorta. The dissection is then carried on to the inferior aspect of the pancreatic neck, in order to achieve a complete mobilisation of the third duodenal portion.

This approach allows the complete control of the SMA area - the inferior vena cava and the left renal vein, crossed by the SMA, are also exposed (Photos 1.5-1.10). Invasion of the SMA can thus be identified at an early stage of the procedure, preventing a useless macroscopic positive resection (R2). Furthermore, this approach allows easier identification and sparing of anatomical variants of the hepatic artery such as a right hepatic artery or a common hepatic artery originating from the SMA, which have 

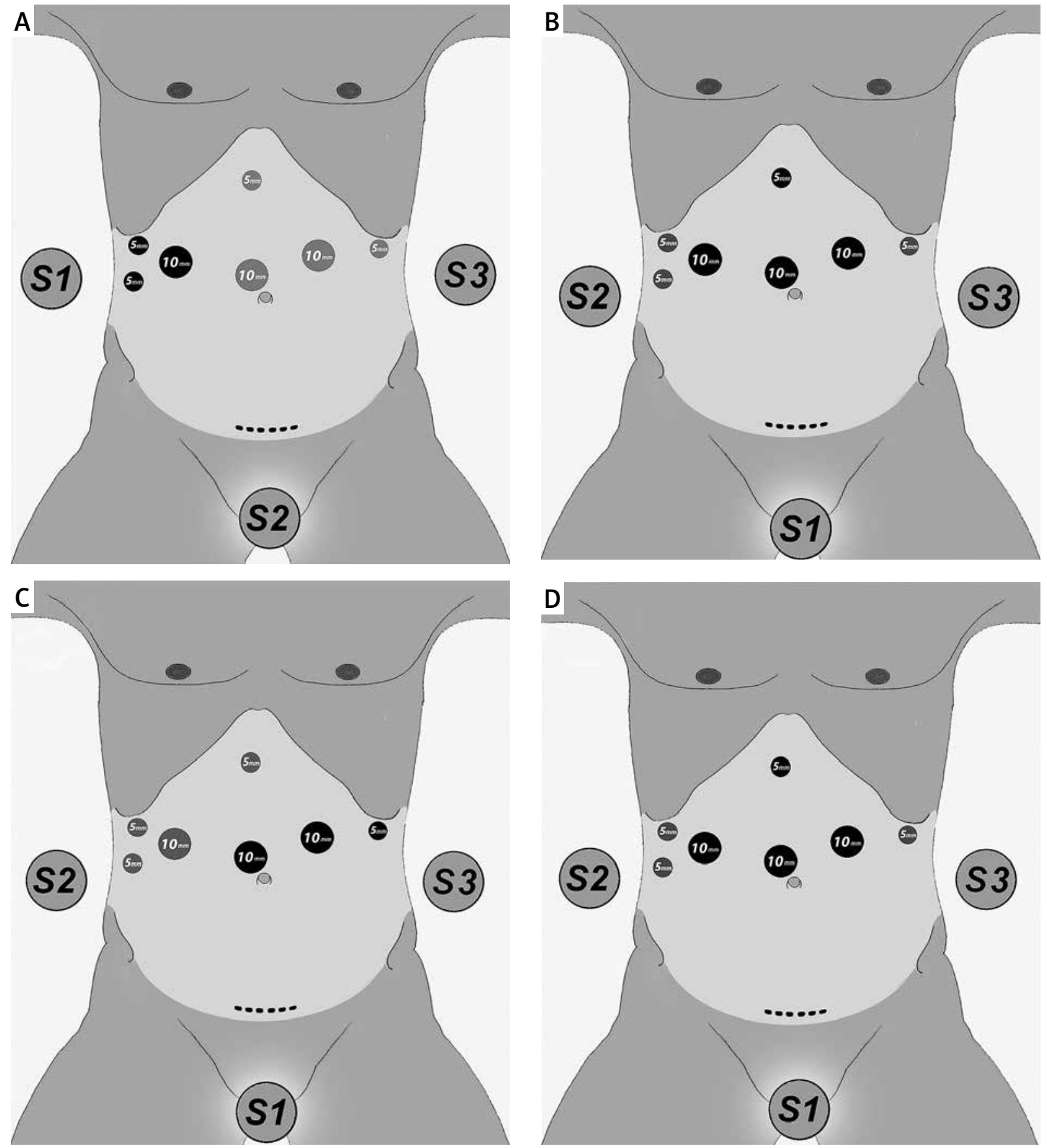

Figure 1. Operative team and trocar placement according to the operative stage. $\mathbf{A}-$ Kocher manoeuvre and AFA. B - Vascular dissection and pancreatic transaction. C - Treitz and uncinate process dissection. D - Biliary section and reconstruction. S1 - first surgeon; S2, S3 - assistants. The black dots represent the trocars used by the first surgeon for each stage of the procedure

to be carefully assessed during the preoperative radiologic study.

The nodal involvement is also assessed during this same operative phase, and in case of macroscopic evidence of nodal metastasis, an extempora- neous nodal sample is sent for fresh-frozen section examination (Photos 1.11-1.12). In fact, the presence of peri-aortic lymph node metastases is considered a contraindication to curative PD, except in the case of neuroendocrine tumours [21, 22]. 

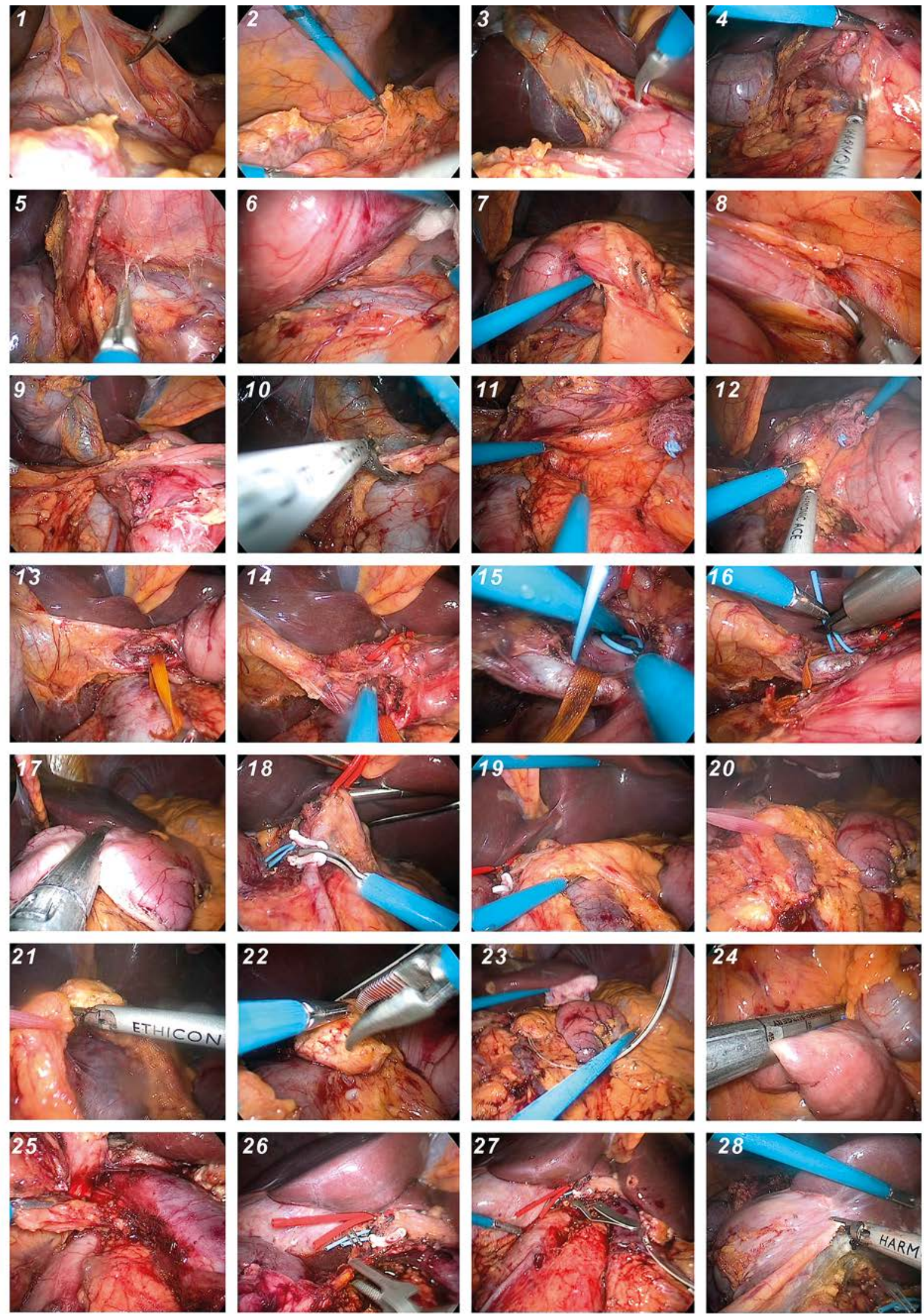

Photo 1. Resection stages of the procedure - explained in text 


\section{Pancreatic transection}

The surgeon ( $\mathrm{S} 1$ ) is positioned between the legs of the patient and his working trocars are placed as shown in Figure $1 \mathrm{~B}$.

During the dissection of the hepatic pedicle, the common bile duct (Photo 1.13), the hepatic artery (Photo 1.14) and the portal vein are identified and isolated (Photos 1.15-1.16). A complete lymphadenectomy of the common hepatic artery, including station 12A, is carried out.

The pylorus is mobilized after the right gastroepiploic vessels and the pyloric artery is sectioned.

The stomach is then transected $2 \mathrm{~cm}$ proximally to the pylorus, using an articulated $45 \mathrm{~mm}$ stapler with a green cartridge (ECHELON FLEX Ethicon Endo-Surgery, Inc. - Cincinnati, OH, USA) (Photo 1.17).

The gastro-duodenal artery is sectioned after having been clipped with a Hem-o-Lok clip (Weck Surgical Instruments, Teleflex Medical, Durham, NC) (Photo 1.18).

The identification of Henle's trunk allows to identify the superior mesenteric vein (SMV), which is dissected, in order to carry out the pancreatic hanging manoeuvre, using a common textile vessel loop (Photos 1.19-1.20).

The pancreatic transection is then performed by use of a harmonic scalpel (Ultracision, Ethicon Endo-Surgery, Inc. - Cincinnati, OH, USA) (Photos 1.21-1.22).

A percutaneous catheter is placed into the distal Wirsung duct in order to evacuate the pancreatic secretion during the rest of the procedure. It will also be used for intraluminal drainage of the pancreaticojejunostomy (Photo 1.23).

\section{Treitz and uncinate process dissection}

The positions of surgeons and the placement of the trocars are shown in Figure $1 \mathrm{C}$.

The Treitz angle is dissected and the first jejunal loop is transected using an articulated $45 \mathrm{~mm}$ stapler with a blue cartridge (ECHELON FLEX Ethicon Endo-Surgery, Inc. - Cincinnati, OH, USA) (Photo 1.24). The duodenum is then retracted caudally.

The retroportal pancreatic lamina is divided from the right side of the superior mesenteric vein (SMV) and the portal vein (PV). The pancreatic head is retracted to the right side of the patient, in order to allow the dissection of the remaining connective tissue attachments of the uncinate process. The portal branches are divided using metal clips and ultrasonic shears (Photo 1.25).

\section{Biliary section}

The surgeon (S1) places himself between the legs of the patient and the trocars are placed as shown in Figure $1 \mathrm{D}$.

The common bile duct is clamped before its section, in order to avoid bile spillage (Photos 1.261.27). Cholecystectomy is performed (Photo 1.28).

\section{Reconstruction}

The greater omentum can be transposed to isolate the anastomoses, allowing to avoid direct contact between the anastomoses and the mesenteric vessels (Photo 2.1).

Finally, the anastomoses are classically performed on the jejunal loop raised through the mesenteric defect, previously created by the dissection of the angle of Treitz (Photos 2.2-2.3):

- the pancreaticojejunostomy is performed in an end-to-side fashion: 4 to 6 simple cardinal stitches of 4/0 PDS are used for the Wirsung. Afterwards, 6 to 8 simples stitches are used to fix the pancreatic and jejunal serosa. The previous catheterization of the Wirsung, by a 3-6 French silicon drain, allows an accurate and targeted suture of the duct (Photos 2.4-2.8);

- the hepaticojejunostomy is performed in an endto-side fashion: around $30 \mathrm{~cm}$ distally to the pancreaticojejunostomy. The posterior side of the anastomosis is done using separate stitches of $4 / 0$ PDS; a running suture is performed on the anterior aspect of the anastomosis (Photos 2.9-2.12);

- the gastrojejunostomy is performed in a side-toside fashion using mechanical suture: using an articulated $45 \mathrm{~mm}$ stapler with a green cartridge (ECHELON FLEX Ethicon Endo-Surgery, Inc. - Cincinnati, $\mathrm{OH}, \mathrm{USA}$ ) and manually placing a running suture (V-Loc 3/0, Covidien, Inc, Mansfield, Massachusetts, USA) to close the gastrojejunostomy (Photos 2.13-2.16).

Drainage is used systematically by means of two Penrose drains (Photos 2.17-2.18) and the specimen is extracted through a Pfannenstiel incision (Photos 2.19-2.20). Two expert surgeons performed all of these procedures, in "tandem" fashion: the resection was carried out by one and the reconstruction by the other. 

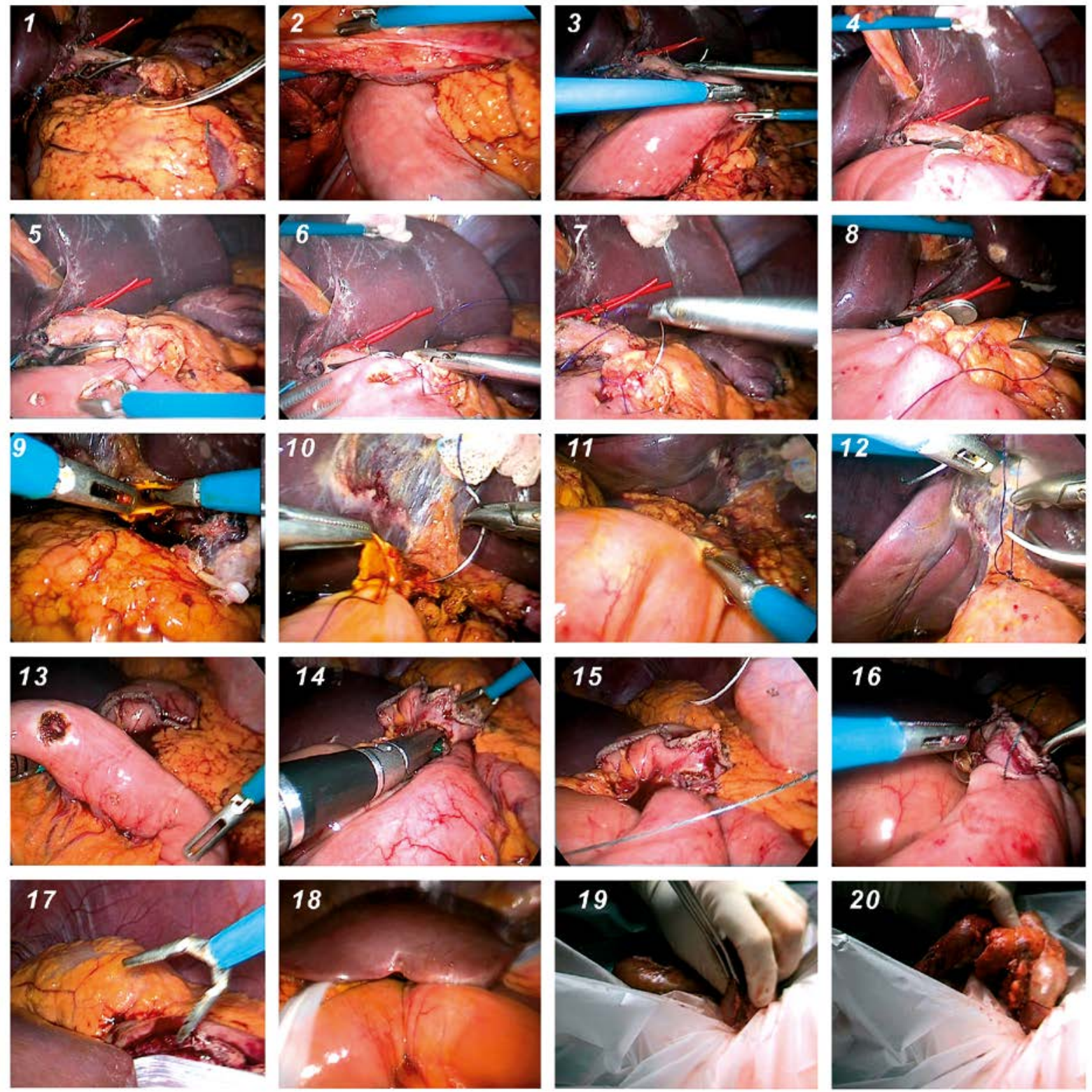

Photo 2. Reconstruction stages of the procedure - explained in text

\section{Results}

The demographic data, operative and post-operative results of the series are summarised in Table I.

Three patients were excluded from the series, initially composed of 15 patients, due to the intraoperative detection of liver metastases in 2 cases and due to extended vascular infiltration of the SMA in one case, not previously described on the preoperative CT scan.

No open conversion, or haemorrhage requiring blood transfusions, was observed in this series.
Concerning the vascular variants, the right hepatic artery originated from the SMA in only one patient, which was consistent with the preoperative CT-scan description. There was no mortality at the $3^{\text {rd }}$ post-operative month (POM). There were three complications: two grade A pancreatic fistulas according to the ISGPF classification and one biliary fistula [19].

Five patients developed a DGE: 2 grade $B$ and 3 grade $A$, according to the ISGPS classification [20]. All complications were resolved conservative- 
Table I. Summary of demographic, operative and post-operative data of the patients included in the study

\begin{tabular}{|c|c|}
\hline Parameter & Results \\
\hline \multicolumn{2}{|l|}{ Demographic data: } \\
\hline Patients, $n$ & 12 \\
\hline Male/female gender, $n$ & $5 / 7$ \\
\hline Age, mean (range) [years] & $62(55-78)$ \\
\hline BMI, mean (range) $\left[\mathrm{kg} / \mathrm{m}^{2}\right]$ & $26(23-30)$ \\
\hline \multicolumn{2}{|l|}{ Operative data: } \\
\hline Operative time, mean (range) [min] & $300(250-540)$ \\
\hline Estimated blood loss, mean (range) [ml] & $180(100-600)$ \\
\hline No. of lymph nodes retrieved (range) & $17(8-31)$ \\
\hline \multicolumn{2}{|l|}{ Post-operative data: } \\
\hline Hospital stay, mean (range) [day] & $18(8-42)$ \\
\hline Mortality at $3^{\text {rd }}$ post-operative month (\%) & 0 \\
\hline \multicolumn{2}{|l|}{ Complications: } \\
\hline $\begin{array}{l}\text { Pancreatic fistula } \\
\text { Biliary fistula }\end{array}$ & $\begin{array}{l}2 \text { patients, (grade } A \text { ) } \\
1 \text { patient }\end{array}$ \\
\hline Blood transfusion & 0 \\
\hline Port site metastasis & $0 / 0$ \\
\hline $\begin{array}{l}\text { Re-intervention at } 3^{\text {rd }} \text { post-operative } \\
\text { month }\end{array}$ & 0 \\
\hline
\end{tabular}

ly and did not require surgical intervention or any endoscopic or radiological interventional procedures.

The histological findings are shown in Table II.

All patients underwent a whole body scan in the $3^{\text {rd }}$ POM. None of them developed local recurrence at this time. No port site metastases were observed. No internal or trocar hernias were reported.

\section{Discussion}

Despite conflicting opinions regarding LPD in the beginning, this procedure has gained increasing acceptance during the last decade [2, 23].

The increasing number of LPD recently reported in the world attests to the safety, the effectiveness and the benefits of laparoscopy, even in terms of oncological outcomes [24-26].
Table II. Summary of pathology findings in the resection specimens

\begin{tabular}{|lc|}
\hline Diagnosis & No. of cases \\
\hline Pancreatic ductal adenocarcinoma & 9 \\
\hline Neuroendocrine carcinoma & 1 \\
\hline Metastatic renal cell carcinoma & 1 \\
\hline Duodenal adenocarcinoma & 1 \\
\hline Specimen characteristics: & $3(1.5-7)$ \\
\hline Tumour size, mean (range) $[\mathrm{cm}]$ & $9(75)$ \\
\hline Ro resection, $n$ (\%) & $7(58)$ \\
\hline Regional lymph node metastases, $n(\%)$ & \\
\hline
\end{tabular}

When compared to open surgery, the operative time is the only factor that is in favour of laparotomy. In contrast, intraoperative blood loss, transfusion need, length of hospital stay and intensive care unit (ICU) stay, and wound infection rate all show significant differences favouring LPD [24].

However, a great number of publications have been reporting significant differences in technicality and surgical approach, limiting the standardization and the transmission of the technique.

Considering that surgical exploration plays a key role for the final assessment of resectability, our team adopted the laparoscopic AFA, which also allows safe vascular control and provides minor surgical invasiveness for unresectable tumours, with the following advantages:

- it allows dissection of the SMA by a posterior approach, and exploration of the SMV and PV confluent, with the well-known benefits of laparoscopic magnification of the field of view;

- it allows a total resection of the retro-pancreatic tissue, performing the dissection from the right to the left and from the back to the front;

- it facilitates the dissection and the sparing of an aberrant hepatic artery, both right and common, coming from the SMA, which should be previously detected on triple-phase CT assessment;

- it allows the resection to be performed only when the oncological assessment is completed, sparing an unnecessary laparotomy.

Finally, the stressful effort of the surgeon, due to the long operative time, was avoided in our study by using the "tandem team" concept: two different surgeons performing resection and reconstruction, which had no negative impact in our series. 


\section{Conclusions}

Laparoscopic pancreatoduodenectomy is feasible and adequate in terms of oncological outcomes. It may be performed in selected patients, by highly skilled laparoscopic teams, with a wide experience in pancreatic surgery.

Pure laparoscopic pancreatoduodenectomy with the AFA could increase the advantage of laparoscopy, allowing early control of vascular structures, avoiding unnecessary resections and bleeding complications.

Further investigation is necessary to obtain a wide awareness of the benefits of PLPD and to clarify the selection criteria of the patients.

\section{Conflict of interest}

The authors declare no conflict of interest.

\section{References}

1. Gagner M, Pomp A. Laparoscopic pylorus-preserving pancreatoduodenectomy. Surg Endosc 1994; 8: 408-10.

2. Palanivelu C, Rajan PS, Rangarajan M, et al. Evolution in techniques of laparoscopic pancreaticoduodenectomy: a decade long experience from a tertiary center. J Hepatobiliary Pancreat Surg 2009; 16: 731-40.

3. Gumbs AA, Rodriguez Rivera AM, Milone L, et al. Laparoscopic pancreatoduodenectomy: a review of 285 published cases. Ann Surg Oncol 2011; 18: 1335-41.

4. Gagner M, Palermo M. Laparoscopic Whipple procedure: review of the literature. J Hepatobiliary Pancreat Surg 2009; 16: 726-30.

5. Zureikat AH, Breaux JA, Steel JL, et al. Laparoscopic pancreaticoduodenectomy be safely implemented? I Gastrointest Surg 2011; 15: 1151-7.

6. Nakao A, Takeda S, Inoue S, et al. Indication and techniques of extended resection for pancreatic cancer. World I Surg 2006; 30: $976-82$

7. Amano H, Miura F, Toyato N, et al. Is pancreatectomy with arterial reconstruction a safe and useful procedure for locally advanced pancreatic cancer. J Hepatobiliary Pancreat Surg 2009; 16: 850-7.

8. Muniraj T, Barve P. Laparoscopic staging and surgical treatment of pancreatic cancer. N Am J Med Sci 2013; 5: 1-9.

9. Urbach DR, Swanstrom LL, Hansen PD. The effect of laparoscopy on survival in pancreatic cancer. Arch Surg 2002; 137: 191-9.

10. Winner M, Allendorf JD, Saif MW. An update on surgical staging of patients with pancreatic cancer highlights from the "2012 ASCO Gastrointestinal Cancers Symposium". San Francisco, CA, USA. J Pancreas (Online) 2012; 10: 13: 143-6.

11. D’Angelica M, Fong Y, Weber S, et al. The role of staging laparoscopy in hepatobiliary malignancy: prospective analysis of 401 cases. Ann Surg Oncol 2003; 10: 183-9.
12. Partensky C. Abord premier de l'artère mésentérique supérieure au cours de la duodénopancréatectomie céphalique. J Chir 2008; 145: 598-600.

13. Sanjay P, Takaori K, Govil S, et al. 'Artery-first' approaches to pancreatoduodenectomy. Br J Surg 2012; 99: 1027-35.

14. Dumitrascu T, David L, Popescu I. Posterior versus standard approach in pancreatoduodenectomy: case-match study. Langenbecks Arch Surg 2010; 395: 677-84.

15. Figueras J, Albiol M, Lopez-Ben S, et al. Cephalic duodenopancreatectomy in periampullary tumours. Dissection of the superior mesenteric artery as in initial approach. Description of the technique and assessment of our experience. Cir Esp 2008; 83: 186-93.

16. Shrikhande SV, Barreto SG, Bodhankar YD, et al. Superior mesenteric artery first combined with uncinate process approach versus uncinate process first approach in pancreatoduodenectomy: a comparative study evaluating perioperative outcomes. Langenbeck's Archives of Surgery 2011; 396: 1205-12.

17. Lupaşcu C, Moldovanu R, Andronic D, et al. Posterior approach pancreaticoduodenectomy: best option for hepatic artery anatomical variants. Hepatogastroenterology 2011; 58: 2112-4.

18. Rose JB, Rocha F, Alseidi A, Helton S. Posterior 'superior mesenteric artery first' approach for resection of locally advanced pancreatic cancer. Ann Surg Oncol 2014; 21: 1927-8.

19. Pratt WB, Maithel SK, Vanounou T, et al. Clinical and economic validation of the International Study Group of Pancreatic Fistula (ISGPF) classification scheme. Ann Surg 2007; 245: 443-51.

20. Wente MN, Bassi C, Dervenis C, et al. Delayed gastric emptying (DGE) after pancreatic surgery: a suggested definition by the International Study Group of Pancreatic Surgery (ISGPS). Surgery 2007; 142: 761-8.

21. Shimada K, Sakamoto Y, Sano T, et al. The role of paraaortic lymph node involvement on early recurrence and survival after macroscopic curative resection with extended lymphadenectomy for pancreatic carcinoma. J Am Coll Surg 2006; 203: 345-52.

22. Yoshida T, Matsumoto T, Sasaki A, et al. Outcome of paraaortic node-positive pancreatic head and bile duct adenocarcinoma. Am J Surg 2004; 187: 736-40.

23. Azagra-Soria JS, Facy O, Goergen $M$, et al. Minimally invasive pancreatic resection. eWJMIS 2012; 2: 28-31.

24. Nakamura M, Nakashima H. Laparoscopic distal pancreatectomy and pancreatoduodenectomy: is it worthwhile? A metaanalysis of laparoscopic pancreatectomy. J Hepatobiliary Pancreat Sci 2013; 20: 421-8.

25. Pericleous S, Middleton N, McKay SC, et al. Systematic review and meta-analysis of case-matched studies comparing open and laparoscopic distal pancreatectomy: is it a safe procedure? Pancreas 2012; 41: 993-1000.

26. Kendrick ML, Cusati D. Total laparoscopic pancreaticoduodenectomy. Arch Surg 2010; 145: 19-23.

Received: 29.04.2015, accepted: 15.07.2015. 